\title{
The Relationship between Methicillin Resistance and Biofilm Composition in Staphylococcus Aureus
}

\author{
Wienclaw Trevor $\mathbf{M}$ and Berges Bradford $\mathbf{K}^{*}$ \\ Department of Microbiology and Molecular Biology, Brigham Young University, USA
}

*Corresponding author: Berges Bradford K, Department of Microbiology and Molecular Biology, Brigham Young University, Provo, UT 84602, USA.

To Cite This Article: Wienclaw Trevor M, Wienclaw Trevor M. The Relationship between Methicillin Resistance and Biofilm Composition in Staphylococcus Aureus. 2020 - 10(1). AJBSR.MS.ID.001464. DOI: 10.34297/AJBSR.2020.10.001464.

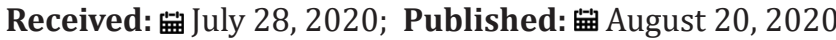

\begin{abstract}
Staphylococcus aureus (SA) is a common commensal bacterium and opportunistic pathogen in humans. Antibiotic-resistant SA strains, such as methicillin-resistant Staphylococcus aureus (MRSA), have become increasingly prevalent in recent years. Another aspect of SA that makes it a successful pathogen is its ability to form biofilms. SA biofilms aid in the invasion of host tissues, and biofilms can form on the surfaces of implanted medical devices, leading to serious, persistent, and sometimes life-threatening infections. Biofilms also prevent effective antibiotic treatment and promote evasion of the host immune response. The biofilms formed by MRSA and by methicillin-susceptible SA (MSSA) are typically quite different, with MSSA biofilms usually composed of polysaccharides and MRSA biofilms typically composed of extracellular proteins and DNA. Interestingly, transfer of the mecA gene (confers resistance to methicillin) alone to a MSSA strain can result in a profound change in biofilm composition to reflect a MRSA biofilm phenotype. The mechanism for this switch in biofilm phenotypes is still an area of active investigation and may yield insights into how to combat this important human pathogen.
\end{abstract}

\section{Introduction}

Staphylococcus aureus (SA) establishes long-term colonization in $20-30 \%$ of the human population, and transient colonization in $60 \%$ [1-3]. Though carriage is usually asymptomatic, infection can cause a wide range of diseases including skin and soft tissue infection, bacteremia, pneumonia, and endocarditis [2,4-6]. SA is possibly the most common cause of food poisoning and the leading cause of death of any infectious agent in the United States of America and is a leading cause of hospital-associated infection throughout the developed world, being second only to Clostridium difficile in the United States [7-9]. Additionally, SA infection rates have increased in recent decades, including both hospital and community acquired infections [10]. Soft-tissue infections are estimated at 48.1 cases per 1,000 population [10]. Infections associated with SA can have mortality rates as high as $25 \%$ [11].

\section{Virulence Factors and Antibiotic Resistance in SA}

SA can carry a variety of virulence factors including leukocidins, hemolysins, enterotoxins, the super antigen TSST-1, protein A, and biofilm genes [6,12-15]. Antibiotic-resistant strains of SA were first detected shortly after the initial use of penicillin [2]. These first resistant strains produced a penicillinase and were still susceptible to the second-generation penicillins, such as methicillin, that were introduced in the early 1960s. However, resistance to these new drugs was reported within one year $[2,8]$. These strains of methicillin-resistant SA (MRSA) had acquired the mecA gene, which encodes an antibiotic-resistant transpeptidase which allows cell wall synthesis to carry on in the presence of beta-lactam antibiotics $[8,16,17]$. Though methicillin is no longer used clinically, the term MRSA is still used to describe any SA strain which carries mecA. MRSA, while originally only found in hospitals, was found to be common in the community outside of hospitals in the 1980s, leading to the terms hospital-acquired MRSA (HA-MRSA) and communityacquired MRSA (CA-MRSA) to differentiate these genetically distinct families of MRSA [4,7]. The antibiotic vancomycin is used to treat MRSA infections as a last resort, though vancomycin-resistant SA (VRSA) strains have been reported in recent years [18,19]. Most clinical SA infections are transmitted via person-to-person contact, 
in either hospital-acquired or community-acquired transmissions $[4,20,21]$. However, SA can also be transmitted to humans from direct contact with living livestock or through exposure to contaminated meats [22-25]. Our recent work has shown that SA isolated from meat products originating in animals fed antibiotics is significantly more resistant to multiple antibiotics compared to SA from meats obtained from animals raised in an antibiotic-free environment [26].

The antibiotic resistance gene of MRSA, mecA, was acquired as part of a mobile genetic element called the staphylococcal cassette chromosome mec (SCCmec). SCCmec is thought to have originated from a non-staphylococcal source, and twelve SCCmec variations have been described [27-29]. In addition to mecA, the SCCmec contains several genes of unknown function and what is known as the $c c r$ gene complex, $c c r A B$ and/or $c c r C$, all of which have roles in promoting site-specific recombination [27]. The gene mecA encodes the membrane-bound transpeptidase penicillin-binding protein 2A (PBP2a) which catalyzes peptidoglycan crosslinking during cell-wall synthesis [16]. This class of enzymes, which were named for their affinity for penicillin, are inhibited when bound by beta-lactams. PBP2a, however, has a uniquely lower affinity for beta-lactams, allowing PBP2a to carry on cell wall synthesis when the activity of the four native SA penicillin-binding proteins is blocked $[28,30]$.

Some SCCmec types have a regulatory system in place consisting of a transcriptional repressor, a sensor-inducer, and an antirepressor to control the expression of mecA, though most clinical MRSA strains appear to have a non-functional regulatory system [31]. High constitutive expression appears to be necessary to give the beta-lactam resistant phenotype associated with MRSA. Because of the slow response time of a functional regulatory system, strains without constitutive expression often appear susceptible to oxacillin in testing, even though they have a functional mecA gene [31,32]. The varied levels of beta-lactam resistance among MRSA strains is due to varied levels of mecA expression [5]. MRSAs can be classified into two categories based on resistance levels: heterogeneously resistant (HeR) and homogeneously resistant (HoR). HeR strains are those able to grow in oxacillin concentrations between 2 and $100 \mu \mathrm{g} / \mathrm{mL}$ while HoR strains can grow in oxacillin concentrations in excess of $100 \mu \mathrm{g} / \mathrm{mL}$ [5].

\section{Biofilm formation by SA}

SA forms robust biofilms, which are communities of cells that can be protected from antibiotic treatment and/or immune cell and immune factors. The cells in a biofilm are surrounded by an extracellular matrix - a network of biologically produced substances that hold the cells together and help them attach to surfaces [33]. SA biofilms are sticky conglomerations of cells surrounded by an extracellular matrix which provide protection from mechanical removal of cells, host immune responses (both innate and adaptive), and antibiotics, giving as much as a 6-log increase in cell viability over planktonic cells following antibiotic challenge [34-36]. Additionally, SA biofilm formation greatly increases the occurrence of horizontal gene transfer, contributing to the spread of antibiotic resistance [37]. SA biofilms are a major concern in hospitals, not just for the danger of infection of damaged host tissue, but also because of the ability of SA to form biofilms on implanted medical devices such as catheters, pacemakers, artificial heart valves, intravascular lines, and joint replacements [38]. Colonization of such devices can lead to serious, chronic infections that are difficult to treat $[11,34]$.

SA biofilm formation is a highly organized process allowing for the formation of complex three-dimensional structures with channels that allow for the flow of nutrients to cells located deeper within the matrix $[34,39]$. The biofilms have tightly regulated growth patterns that regulate attachment to a surface, the growth and expansion of the biofilm, and detachment and spread to other sites $[34,36]$. These processes are regulated through quorum sensing, allowing the optimal cell density to be maintained by regulating the dispersal of cells for spread to new areas. During the growth phase of biofilms some cells will even undergo an apparently altruistic autolysis to provide neighboring cells with the materials necessary to construct the extracellular matrix, such as DNA $[39,40]$.

Though there are some general characteristics shared amongst SA biofilms, the composition of the extracellular matrix from strain to strain can be drastically different $[38,41,42]$. In general, these varied extracellular matrix compositions are categorized into two classes based upon the presence of polysaccharide intercellular adhesin (PIA), forming both PIA-dependent and PIA-independent biofilms [5,38]. PIA-independent biofilms tend to have a matrix composed primarily of protein and extracellular DNA/eDNA. The biofilm class of any particular strain can be determined by a series of simple tests. Biofilms are grown in 96 well plates and then treated with proteinase $\mathrm{K}$ to degrade extracellular proteins; DNase to degrade eDNA; or sodium metaperiodate which oxidizes polysaccharide linkages. PIA-dependent biofilms are unaffected by proteinase $\mathrm{K}$ treatment and dispersed by sodium metaperiodate treatment, while PIA-independent biofilms are dispersed by proteinase $\mathrm{K}$ treatment or DNase and are unaffected by sodium metaperiodate $[41,42]$. While there have been thorough studies of the composition and genes associated with each class of biofilm [43-45] only an overview of the major components will be given here.

\section{PIA-dependent SA biofilms}

PIA-dependent biofilms are the classic biofilm type; they were the first studied and are what are usually described about 
typical S. aureus strains $[11,44,46]$. Their extracellular matrix consists primarily of PIA, built from the polysaccharide poly$\beta(1-6)-\mathrm{N}$-acetylglucosamine, but it may also contain a variety of proteins, extracellular DNA (eDNA), and amyloid fibrils [44]. Many cytoplasmic proteins and genomic DNA may become associated with the extracellular matrix as cells undergo autolysis. This altruistic act of some cells, which is triggered through quorum sensing, provides the raw materials necessary to form the biofilm $[34,39,40]$. The eDNA, while not necessary for the structural integrity of PIA-dependent biofilms, is important for the formation of amyloid fibrils from phenol-soluble modulins, which contribute to biofilm stability $[42,47]$. The primary component of PIA-dependent biofilms is, of course, PIA. This polysaccharide is produced and assembled into the extracellular matrix by the products of the icaADBC operon. icaA encodes an $\mathrm{N}$-acetylglucosaminyltransferase that synthesizes PIA; icaD produces a product that, while not fully understood, is known to increase the efficiency of icaA; icaB produces an N-deacetylase which partially deacetylates PIA; and icaC is involved in the exportation of PIA to the cell surface $[44,48]$. While several genes are known to influence the production of PIA the best characterized is icaR, a divergently transcribed repressor of the ica operon located just upstream of the icaA gene [48-50].

\section{PIA-independent SA biofilms}

PIA-independent biofilms are most notably characterized by the lack of PIA. These biofilms rely solely on extracellular proteins and eDNA for their structural integrity, a difference which can be seen by electron microscopy [38,41]. The reliance of eDNA in PIAindependent biofilms makes the agr quorum-sensing system, which triggers autolysis, vital in biofilm formation [40,51]. Once DNA is released it is thought to interact with cell surface proteins to bind cells one to another [38]. The primary proteins involved in the PIAindependent biofilm type are the membrane-bound fibronectinbinding proteins FnBPA and FnBPB [40]. The function of these proteins in biofilm formation appears to be redundant as either may be knocked out and biofilms will continue to form normally [52]. Other membrane-bound proteins, such as protein A and SasG have been shown to be involved in PIA-independent biofilm formation, though their specific functions and possible interactions with eDNA have yet to be studied $[38,43]$. Extracellular proteases are of crucial importance for PIA-independent biofilms. Protease production is limited during biofilm maturation but increased for biofilm dispersal to degrade the extracellular matrix and release the cells $[35,51,53]$.

\section{The link between antibiotic resistance and biofilm type in SA}

Both MRSA and MSSA produce biofilms, but the biofilms they produce have been reported to be inherently different [38]. The only definite difference between a MRSA strain and a MSSA strain is the presence of the antibiotic resistance gene mecA. High-level expression of the mecA gene has been shown to repress the ica operon which is necessary for biofilm polysaccharide production [41], but the mechanism by which this occurs is unknown. In addition, the quorum-sensing agr system which is an important part of biofilm maturation and dispersion can also repress by mecA.

It has been observed that MRSA strains tend to produce a PIA-independent biofilm type while MSSA tend to produce PIA-dependent biofilms [5,38,41,43,54]. This trend is typically described in the context of homogeneously resistant (HoR) types of MRSA strains [5,38]. Pozzi et al transformed MSSA cells known to produce a PIA-dependent biofilm with a plasmid containing mecA. These cells were then put through a selection to isolate a HoR MRSA strain. A complete shift in biofilm type from PIA-dependent to PIA-independent was observed and icaA expression was drastically reduced. The plasmid was then cured (reverting back to MSSA) and the strain returned to a PIA-dependent biofilm type and ica A expression returned to normal. How this shift in biofilm type is accomplished is unknown. PBP2a (encoded by mecA) is membrane-bound and is not known to have any direct effect on transcription, yet mutation of its active site abolishes the effects on ica transcription. Furthermore, the repression of the ica operon was found to be icaR independent. It has been suggested that a change in cell wall architecture through the action of PBP2a may be responsible for this drastic shift in biofilm composition, yet this is still uncertain and more research into these mechanisms has yet to be done $[5,38]$.

Biofilms are known to be hotspots for horizontal gene transfer (HGT). While biofilms likely contribute to antibiotic resistance gene acquisition in SA as well, this topic has not been explored much in the literature. Since eDNA is more common in MRSA biofilms as compared to MSSA biofilms, it is possible that MRSA biofilms have higher rates of HGT than MSSA biofilms. If true, this could potentially explain a mechanism by which MRSA has gained such high levels of antibiotic resistance.

\section{Future perspective}

The PIA-dependent biofilms typically seen in MSSA strains appear to be thicker and less penetrable than the PIA-independent biofilms seen with MRSA strains [38]. In our recent study, we showed that MSSA biofilms were not as affected by vancomycin, silver nanoparticles, or a combinatorial treatment as compared to MRSA biofilms [55]. Perhaps MSSA strains have evolved to produce a more robust biofilm which is less penetrable to antibiotics, since these isolates are inherently more susceptible to antibiotics. However, this hypothesis fails to explain why MRSA isolates would produce a less robust biofilm since immune pressure is likely similar for both MRSA and MSSA. It is possible that SA strains that develop both high-level antibiotic resistances combined with 
strong/PIA-dependent biofilm formation are too virulent, and that natural selection prevents their widespread formation.

A better understanding of the control mechanisms of biofilm formation may allow for the discovery of new ways to disperse biofilms, and to promote weaker biofilm formation which may allow for more effective antibiotic therapy of SA infections.

\section{References}

1. von Eiff C, Becker K, Machka K, Stammer H, Peters G (2001) Nasal carriage as a source of Staphylococcus aureus bacteremia. Study Group. N Engl J Med 344(1): 11-16.

2. Monecke S, Coombs G, Shore AC, Coleman DC, Akpaka P, et al. (2011) a field guide to pandemic, epidemic and sporadic clones of methicillinresistant Staphylococcus aureus. PLoS One 6(4): e17936.

3. Balasubramanian D, Harper L, Shopsin B, Torres VJ (2017) Staphylococcus aureus pathogenesis in diverse host environments. Pathog Dis 75(1): ftx005.

4. Sowash MG, Uhlemann AC (2014) Community-associated methicillinresistant Staphylococcus aureus case studies. Methods Mol Biol 1085: 25-69.

5. Pozzi C, Waters EM, Rudkin JK, Schaeffer CR, Lohan AJ, et al. (2012) Methicillin resistance alters the biofilm phenotype and attenuates virulence in Staphylococcus aureus device-associated infections. PLoS Pathog 8(4): e1002626.

6. Van Hal SJ, Jensen SO, Vaska VL, Espedido BA, Paterson DL, et al. (2012) Predictors of mortality in Staphylococcus aureus Bacteremia. Clin Microbiol Rev 25(2): 362-386.

7. DeLeo FR, Chambers HF (2009) Reemergence of antibiotic-resistant Staphylococcus aureus in the genomics era. J Clin Invest 119(9): 2464 2474 .

8. Kobayashi SD, Malachowa N, DeLeo FR (2015) Pathogenesis of Staphylococcus aureus abscesses. Am J Pathol 185(6): 1518-1527.

9. Kluytmans JA (2010) Methicillin-resistant Staphylococcus aureus in food products: cause for concern or case for complacency? Clin Microbiol Infect 16(1): 11-15.

10. Tong SY, Davis JS, Eichenberger E, Holland TL, Fowler VG, et al. (2015) Staphylococcus aureus infections: epidemiology, pathophysiology, clinical manifestations, and management. Clin Microbiol Rev 28(3): 603-661.

11. Archer NK, Mazaitis MJ, Costerton JW, Leid JG, Powers ME, et al. (2011) Staphylococcus aureus biofilms: properties, regulation, and roles in human disease. Virulence 2(5): 445-459.

12. Bien J, Sokolova O, Bozko P (2011) Characterization of Virulence Factors of Staphylococcus aureus: Novel Function of Known Virulence Factors That Are Implicated in Activation of Airway Epithelial Proinflammatory Response. J Pathog 2011: 601905.

13. Oogai Y, Matsuo M, Hashimoto M, Kato F, Sugai M, et al. (2011) Expression of virulence factors by Staphylococcus aureus grown in serum. Appl Environ Microbial 77(22): 8097-8105.

14. Löffler B, Hussain M, Grundmeier M, Brück M, Holzinger D, et al. (2010) Staphylococcus aureus panton-valentine leukocidin is a very potent cytotoxic factor for human neutrophils. PLoS Pathog 6(1): e1000715.

15. Watkins RR, David MZ, Salata RA (2012) Current concepts on the virulence mechanisms of meticillin-resistant Staphylococcus aureus. J Med Microbiol 61(Pt 9): 1179-1193.

16. Wu CY, Hoskins J, Blaszczak LC, Preston DA, Skatrud PL, et al. (1992) Construction of a water-soluble form of penicillin-binding protein 2 a from a methicillin-resistant Staphylococcus aureus isolate. Antimicrob Agents Chemother 36(3): 533-539.
17. Ballhausen B, Kriegeskorte A, Schleimer N, Peters G, Becker K, et al. (2014) The mecA homolog mecC confers resistance against $\beta$-lactams in Staphylococcus aureus irrespective of the genetic strain background. Antimicrob Agents Chemother 58(7): 3791-3798.

18. Rossi F, Diaz L, Wollam A, Panesso D, Zhou Y, et al. (2014) Transferable vancomycin resistance in a community-associated MRSA lineage. N Engl J Med 370(16): 1524-1531.

19. Hasan R, Acharjee M, Noor R (2016) Prevalence of vancomycin resistant. Ci Ji Yi Xue Za Zhi 28(2): 49-53.

20. Hughes C, Tunney M, Bradley MC (2013) Infection control strategies for preventing the transmission of meticillin-resistant Staphylococcus aureus (MRSA) in nursing homes for older people. Cochrane Database Syst Rev (11): CD006354.

21. Sergelidis D, Angelidis AS (2017) Methicillin-resistant Staphylococcus aureus: a controversial food-borne pathogen. Lett Appl Microbiol 64(6): 409-418.

22. Lee J H (2003) Methicillin (Oxacillin)-resistant Staphylococcus aureus strains isolated from major food animals and their potential transmission to humans. Appl Environ Microbiol 69(11): 6489-6494.

23. Armand-Lefevre L, Ruimy R, Andremont A (2005) Clonal comparison of Staphylococcus aureus isolates from healthy pig farmers, human controls, and pigs. Emerg Infect Dis 11(5): 711-714.

24. Graveland H, Wagenaar JA, Heesterbeek H, Mevius D, van Duijkeren E, et al. (2010) Methicillin resistant Staphylococcus aureus ST398 in veal calf farming: human MRSA carriage related with animal antimicrobial usage and farm hygiene. PLoS One 5(6): e10990.

25. Juhász-Kaszanyitzky E, Jánosi S, Somogyi P, Dán A, van der Graaf-van Bloois L, et al. (2007) MRSA transmission between cows and humans. Emerg Infect Dis 13(4): 630-632.

26. Haskell KJ, Schriever SR, Fonoimoana KD, Haws B, Hair BB, et al. (2018) Antibiotic resistance is lower in Staphylococcus aureus isolated from antibiotic-free raw meat as compared to conventional raw meat. PLoS One 13(12): e0206712.

27. (IWG-SCC) IWGotCoSCCE (2009) Classification of staphylococcal cassette chromosome mec (SCCmec): guidelines for reporting novel SCCmec elements. Antimicrob Agents Chemother 53(12): 4961-4967.

28. Fishovitz J, Hermoso JA, Chang M, Mobashery S (2014) Penicillinbinding protein $2 \mathrm{a}$ of methicillin-resistant Staphylococcus aureus. IUBMB Life 66(8): 572-577.

29. Monecke S, Jatzwauk L, Müller E, Nitschke H, Pfohl K, et al. (2016) Diversity of SCCmec Elements in Staphylococcus aureus as Observed in South-Eastern Germany. PLoS One 11(9): e0162654.

30. Lim D, Strynadka NC (2002) Structural basis for the beta lactam resistance of PBP2a from methicillin-resistant Staphylococcus aureus. Nat Struct Biol 9(11): 870-876.

31. Arêde P, Milheiriço C, de Lencastre H, Oliveira DC (2012) The antirepressor MecR2 promotes the proteolysis of the mecA repressor and enables optimal expression of $\beta$-lactam resistance in MRSA. PLoS Pathog 8(7): e1002816.

32. Oliveira DC, de Lencastre H (2011) Methicillin-resistance in Staphylococcus aureus is not affected by the overexpression in Trans of the mecA gene repressor: a surprising observation. PLoS One 6(8): e23287.

33. Joo HS, Otto M (2012) Molecular basis of in vivo biofilm formation by bacterial pathogens. Chem Biol 19(12): 1503-1513.

34. Periasamy S, Joo HS, Duong AC, Bach TH, Tan VY, et al. (2012) How Staphylococcus aureus biofilms develop their characteristic structure. Proc Natl Acad Sci U S A 109(4): 1281-1286.

35. Boles BR, Horswill AR (2008) Agr-mediated dispersal of Staphylococcus aureus biofilms. PLoS Pathog 4(4): e1000052. 
36. Moormeier DE, Bayles KW (2017) Staphylococcus aureus biofilm: a complex developmental organism. Mol Microbiol 104(3): 365-376.

37. Savage VJ, Chopra I, O’Neill AJ (2013) Staphylococcus aureus biofilms promote horizontal transfer of antibiotic resistance. Antimicrob Agents Chemother 57(4): 1968-1970.

38. McCarthy H, Rudkin JK, Black NS, Gallagher L, O’Neill E, et al. (2015) Methicillin resistance and the biofilm phenotype in Staphylococcus aureus. Front Cell Infect Microbiol 5: 1 .

39. Lauderdale KJ, Boles BR, Cheung AL, Horswill AR, et al. (2009) Interconnections between Sigma B, agr, and proteolytic activity in Staphylococcus aureus biofilm maturation. Infect Immun 77(4): 16231635.

40. Houston P, Rowe SE, Pozzi C, Waters EM, O'Gara JP, et al. (2011) Essential role for the major autolysin in the fibronectin-binding protein-mediated Staphylococcus aureus biofilm phenotype. Infect Immun 79(3): 1153-1165.

41. O'Neill E, Pozzi C, Houston P, Smyth D, Humphreys H, et al. (2007) Association between methicillin susceptibility and biofilm regulation in Staphylococcus aureus isolates from device-related infections. J Clin Microbiol 45(5): 1379-1388.

42. Mack D, Siemssen N, Laufs R (1992) Parallel induction by glucose of adherence and a polysaccharide antigen specific for plastic-adherent Staphylococcus epidermidis: evidence for functional relation to intercellular adhesion. Infect Immun 60(5): 2048-2057.

43. Boles BR, Thoendel M, Roth AJ, Horswill AR (2010) Identification of genes involved in polysaccharide-independent Staphylococcus aureus biofilm formation. PLoS One 5(4): e10146.

44. Arciola CR, Campoccia D, Ravaioli S, Montanaro L (2015) Polysaccharide intercellular adhesin in biofilm: structural and regulatory aspects. Front Cell Infect Microbiol 5: 7.

45. Tu Quoc PH, Genevaux P, Pajunen M, Savilahti H, Georgopoulos C, et al. (2007) Isolation and characterization of biofilm formation-defective mutants of Staphylococcus aureus. Infect Immun 75(3): 1079-1088.

46. Fluckiger U, Ulrich M, Steinhuber A, Döring G, Mack D, et al. (2005) Biofilm formation, icaADBC transcription, and polysaccharide intercellular adhesin synthesis by staphylococci in a device-related infection model. Infect Immun 73(3): 1811-1819.

47. Schwartz K, Ganesan M, Payne DE, Solomon MJ, Boles BR, et al. (2016) Extracellular DNA facilitates the formation of functional amyloids in Staphylococcus aureus biofilms. Mol Microbiol 99(1): 123-124.

48. Conlon KM, Humphreys H, O'Gara JP (2002) icaR encodes a transcriptional repressor involved in environmental regulation of ica operon expression and biofilm formation in Staphylococcus epidermidis. J Bacteriol 184(16): 4400-4408.

49. Cue D, Lei MG, Luong TT, Kuechenmeister L, Dunman PM, et al. (2009) Rbf promotes biofilm formation by Staphylococcus aureus via repression of icaR, a negative regulator of icaADBC. J Bacterial 191(20): 6363-6373.

50. Cue D, Lei MG, Lee CY (2012) Genetic regulation of the intercellular adhesion locus in staphylococci. Front Cell Infect Microbiol 2: 38.

51. Yarwood JM, Bartels DJ, Volper EM, Greenberg EP (2004) Quorum sensing in Staphylococcus aureus biofilms. J Bacteriol 186(6): 18381850 .

52. O’Neill E, Pozzi C, Houston P, Humphreys H, Robinson DA, et al. (2008) a novel Staphylococcus aureus biofilm phenotype mediated by the fibronectin-binding proteins, FnBPA and FnBPB. J Bacteriol 190(11): 3835-3850.

53. Mootz JM, Malone CL, Shaw LN, Horswill AR (2013) Staphopains modulate Staphylococcus aureus biofilm integrity. Infect Immun 81(9): 3227-3238.

54. Dakheel KH, Abdul Rahim R, Neela VK, Al-Obaidi JR, Hun TG, et al. (2016) Methicillin-Resistant Staphylococcus aureus Biofilms and Their Influence on Bacterial Adhesion and Cohesion. Biomed Res Int 2016: 4708425

55. Hair BB, Conley ME, Wienclaw TM, Conley MJ, Heato n MJ, et al (2018) Synergistic Activity of Silver Nanoparticles and Vancomycin against a Spectrum of Staphylococcus aureus Biofilm Types. Journal of Bacteriology and Mycology 5: 1089. 